\title{
Mapping hydrothermal altered mineral deposits using Landsat 7 ETM+ image in and around Kuju volcano, Kyushu, Japan
}

\author{
Md. Bodruddoza Mia ${ }^{1,2, *}$ and Yasuhiro Fujimitsu ${ }^{3}$ \\ ${ }^{1}$ Department of Earth Resources Engineering, Graduate School of Engineering, Kyushu University, Kyushu, Japan. \\ ${ }^{2}$ Department of Geology, University of Dhaka, Dhaka-1000, Bangladesh. \\ ${ }^{3}$ Department of Earth Resources Engineering, Kyushu University, Kyushu, Japan. \\ ${ }^{*}$ Corresponding author. e-mail: bodruddoza@mine.kyushu-u.ac.jp
}

\begin{abstract}
To evaluate the conventional methods for mapping hydrothermal altered deposits by using Landsat 7 ETM+ image in and around Kuju volcano is the prime target of our study. The Kuju volcano is a mountainous composite which consists of hornblende-andesite lava domes and associated lava flows. We used the colour composite, band ratio, principal component analysis, least square fitting and reference spectra analysis methods. The colour composite and band ratio methods showed very clearly the hydrothermal altered deposits of clay minerals, iron oxides and ferric oxides around the fumaroles. The principal component analysis using the Crosta technique also enabled us to represent undoubtedly the altered hydroxyl and iron-oxide mineral deposits of this region concentrating around the fumaroles. Least square fitting method illustrated the goethite, hematite and clay alteration region. Finally the target detection method for reference spectral analysis by using ENVI 4.3 detected the representative hydrothermal altered minerals around Kuju volcano fumaroles area. Therefore, all the methods showed high efficiency for mapping hydrothermal altered deposits especially iron-oxide minerals such as hematite, goethite and jarosite, which are alteration products of hydrothermal sulfides around the fumaroles.
\end{abstract}

\section{Introduction}

The definition of hydrothermal alteration is the reflection of response of pre-existing, rock-forming minerals to physical and chemical conditions different than those, under which they originally formed, especially by the action of hydrothermal fluids (Beane 1982). The hydrothermal fluid processes alter the mineralogy and chemistry of the host rocks that can produce distinctive mineral assemblages which vary according to the location, degree and duration of those alteration processes. When these alteration products are exposed at the surface, they can be mapped as a zonal pattern. They appear concentrically around a core which has the highest grade alteration and greatest economic interest. The importance of the recognition of such spatial patterns of alteration makes the 'remote sensing technique' one of the standard procedures in exploration geology, due to its high efficiency and low cost (Yetkin 2003).

There are many studies around the world related to hydrothermal alteration mapping using multispectral satellite images especially, Landsat and Aster (e.g., Abdelsalam et al. 2000; Ramadan et al. 2001; Madani et al. 2003; Ramadan and Kontny 2004). Thematic mapping multispectral images from Landsat satellites cover the visible

Keywords. Mapping; hydrothermal alteration; Landsat 7 ETM+; fumaroles; Kuju volcano. 
and infrared spectrum of hydrothermal alterations (Hunt 1979; Hunt and Ashley 1979). The major types of alteration found in volcanic areas are potassic, phyllic, argilic, propylitic, and silicification. Each type of alteration has diagnostic minerals in their respective rocks. Satellite imaging plays an efficient role in differentiating the representative minerals for the different types of alterations. Hydrothermal alteration could also be used to locate faults in any volcanic area. Usually, the alteration zones are correlated with the discharged flows from the volcanic reservoir, and with the main structures that control the permeability of the reservoir and the cap rock. So, locating hydrothermally altered rocks in the surface can help to determine the presence of upflows from the volcanic reservoir.

Previous studies explained the fact that certain minerals associated with hydrothermal processes, such as iron-bearing minerals (e.g., goethite, hematite, jarosite and limonite) and hydroxyl bearing minerals (e.g., kaolinite and K-micas) show diagnostic spectral features that allow their remote identification (Hunt 1980). Iron oxide is quite a common constituent of alteration zones associated with hydrothermal sulphide deposits (Poormirzaee and Oskouei 2009). The major ironoxide species - goethite, jarosite, and hematite that are formed from the weathering of sulfides absorb energy at different frequencies in the VNIR/SWIR (Rowan 1983), providing a means of discrimination using hyperspectral scanners (Taranik et al. 1991). Hydroxyl-bearing minerals form the most widespread product of alteration.

The study area, Kuju volcano $\left(33^{\circ} 05^{\prime} 32.38^{\prime \prime} \mathrm{N}\right.$, $\left.131^{\circ} 14^{\prime} 17.82^{\prime \prime} \mathrm{E}\right)$, lies in Oita Prefecture and is the active center of the Hohi volcanic zone in central Kyushu Island, Japan, where a $70 \times 45 \mathrm{~km}$ volcanotectonic depression has developed under an extensional stress field from $6 \mathrm{Ma}$ (Kamata and Kodama 1994) (figure 1). The last eruption by Kuju volcano occurred on October 11, 1995. Kuju volcano is a mountainous composite volcano which consists of 16 homblende-andesite lava domes and associated lava flows, five pyroxene-andesite cones, and one olivine-basalt cone (Kamata et al. 1996).

The main target of our study is to detect and map the hydrothermal altered minerals in

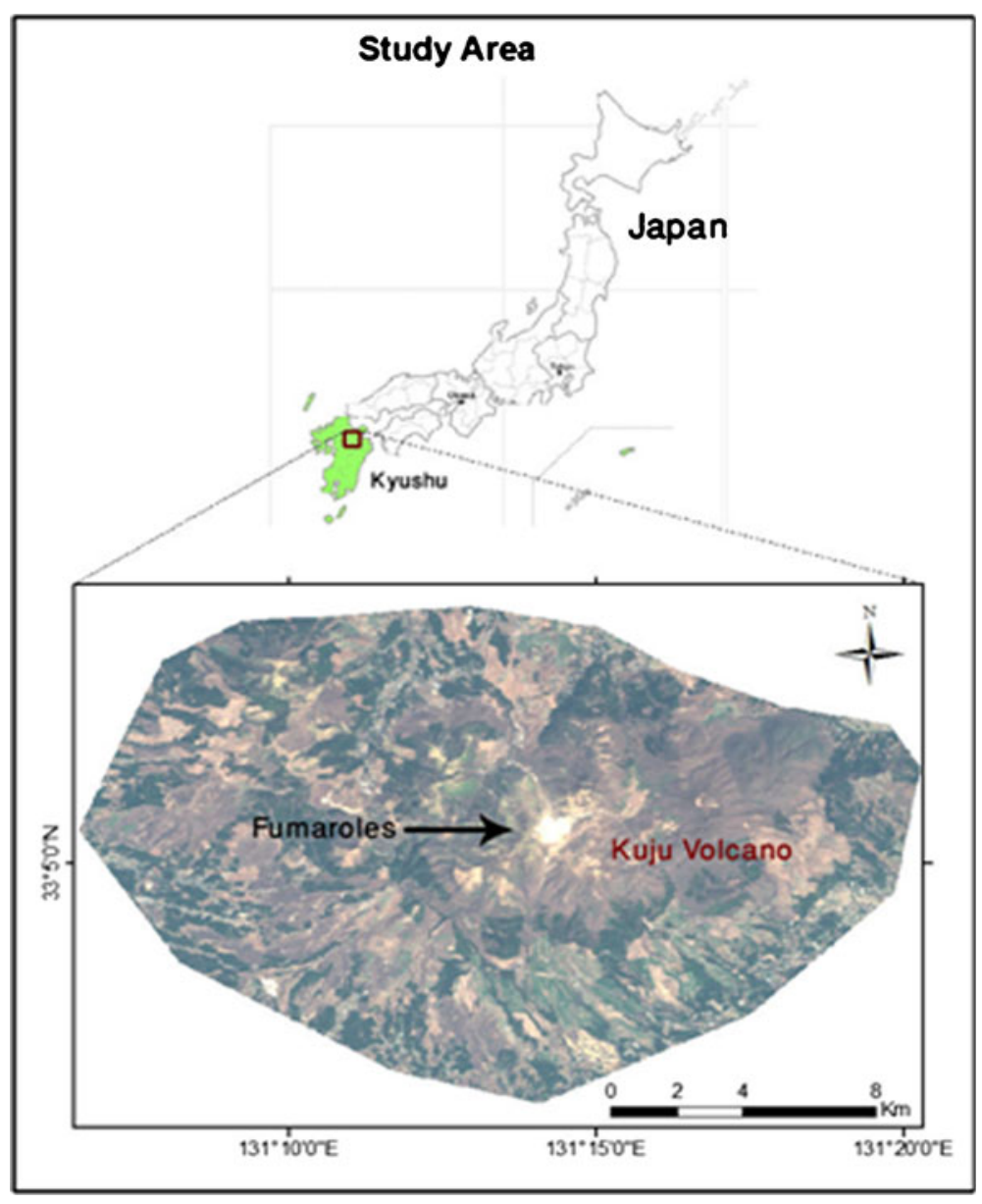

Figure 1. Location map of our study area. 
and around the Kuju volcano area using satellite imaging information by the conventional methods, i.e., colour composites, band ratio, Crosta technique, Ls-fit method and library spectral analysis. In this study, a satellite image was acquired from the Landsat 7 ETM+ on April 17, 2000. This image, titled Landsat-7 Enhanced Thematic Mapper Plus (ETM+) data scenes number 112/37(Path/Row), shows the areas around Kuju volcano as well as most of Oita Prefecture and a part of Kumamoto Prefecture of Kyushu Island in Japan. A reconnaissance field work was carried out around the fumaroles area of Kuju volcano on December 9, 2010. We found that andesitic rocks are exposed in the fumaroles area with hydrothermally sulfide altered minerals.

\section{Satellite image pre-processing}

The 'Enhanced Thematic mapper (ETM) plus' sensor was launched on April 15, 1999 on the Landsat 7 spacecraft. The sensor uses the whiskbroom scanner, common to the Thematic Mapper (TM) sensor family that was flown on Landsats 4 and 5 . The sensor improved with several evolutionary refinements including the addition of a $15-\mathrm{m}$ resolution panchromatic band and a higher resolution $(60 \mathrm{~m})$ thermal band (Markham et al. 2008) (table 1). Another improvement of the ETM+ instrument over the previous TM one, for enhancing radiometric precision, and consequently for better land-cover discrimination, is its capability to scan in either a low or high gain state. Here, gain is denoted as an increase in signal power during its transmission from one point to another in order to maximize the radiometric resolution ( 8 bits in the case of the ETM+) without saturating the detectors (whose digital number (DN) maximum is 255) (Karnieli et al. 2004).

The image processing techniques were adopted here used ENVI 4.3 and ArcGIS 9.3. Raw digital

Table 1. Summary of Landsat 7 ETM+ sensor parameters.

\begin{tabular}{lcc}
\hline Band & $\begin{array}{c}\text { Wavelength range } \\
(\mu \mathrm{m})\end{array}$ & $\begin{array}{c}\text { Pixel size } \\
(\mathrm{m})\end{array}$ \\
\hline 1 & $0.450-0.515$ & 30 \\
2 & $0.525-0.605$ & 30 \\
3 & $0.630-0.690$ & 30 \\
4 & $0.750-0.900$ & 30 \\
5 & $1.55-1.75$ & 30 \\
6 & $10.40-12.50$ & $60(30)$ \\
7 & $2.09-2.35$ & 30 \\
8 & $0.520-0.900$ & 15 \\
\hline
\end{tabular}

satellite data usually includes geometric distortions due to sensor geometry, scanner, platform instabilities, earth rotation, earth curvature, etc., and it is necessary to correct and adapt them (Mather 1987; Lillesand et al. 2004). Geometric correction has been done for the digital data of the ETM+ bands of the study area. The geo-referencing is carried out under the projection system of geographic (lat./long.) and datum WGS-84 using ground control points selected from topographic sheets of scale 1:50,000. The root mean square error (RMS) in the geometric processing was less than one pixel.

\section{Hydrothermal alteration mapping: methods, analysis and results}

\subsection{Colour composites}

Three additive colours (i.e., red, green and blue) were used to display multispectral bands in the colour composite method where the spectral response of the minerals indicates a maximum in their reflectance. This enhancement is achieved by combining bands in the visible and the infrared
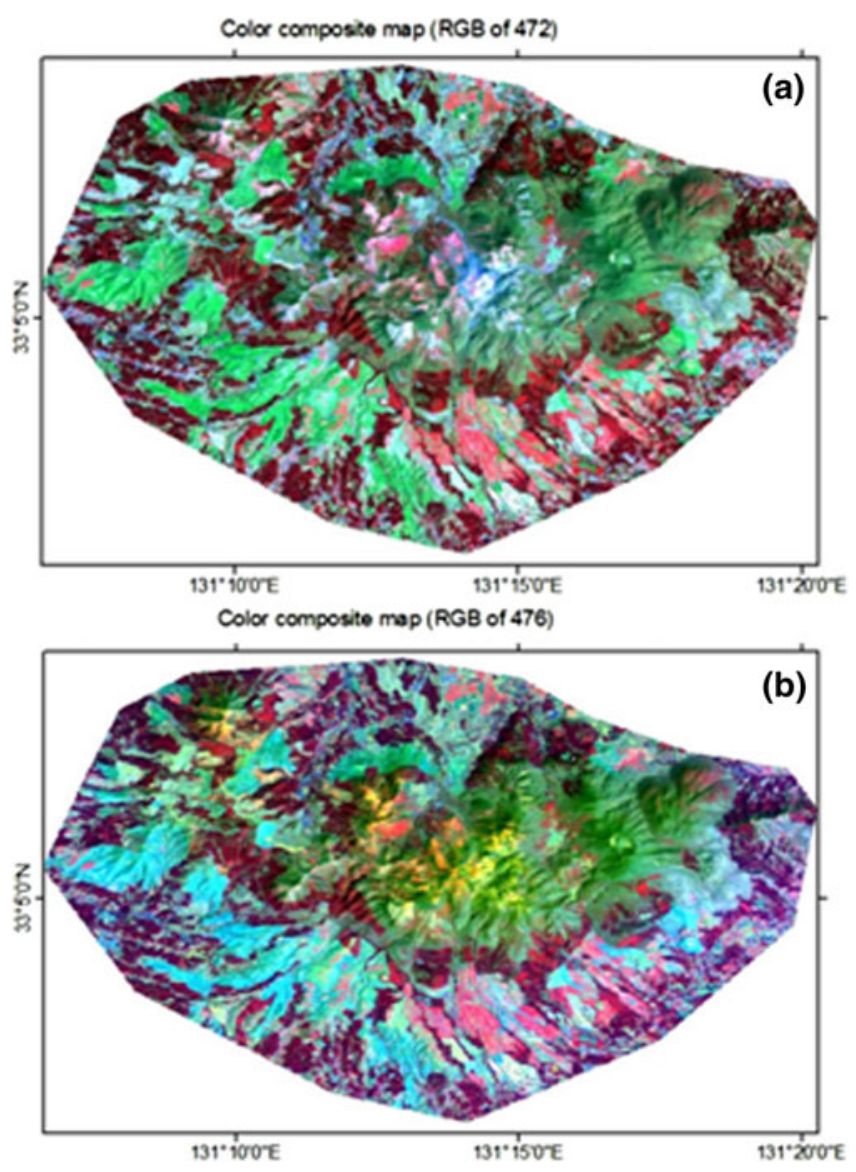

Figure 2. Hydrothermal alteration zone as: (a) deep green and blue (RGB as 4:7:2) and (b) deep green (RGB as 4:7:6). 
portion (Crosta and Moore 1989). Figure 2 illustrated hydrothermal alteration areas showing deep green colour of false colour composites (RGB) of bands 4:7:2 and 4:7:6.

\subsection{Band ratio}

The band ratio is a technique that has been used for many years in remote sensing to display spectral variations effectively (e.g., Goetz et al. 1983). It is based on highlighting the spectral differences that are unique to the materials being mapped. Identical surface materials can give different brightness values because of the topographic slope and aspect, shadows, or seasonal changes in sunlight illumination angle and intensity. These variances affect the viewer's interpretations and may lead to misguided results. Therefore, the band ratio operation could be able to transform the data without reducing the effects of such environmental condition. In addition, ratio operation may also provide unique information that is not available in any single band which is very useful for disintegrating the surface materials (Jensen 1996). The band ratio images are known for enhancement of spectral contrasts among the bands considered in the ratio operation and have successfully been used in mapping of alteration zones (Segal 1983). From the theoretical knowledge of mineral's spectral properties, it is well recognized that the Landsat TM bands ratios of $3 / 1,5 / 7,5 / 4$ are analyzed for iron oxides, hydroxyl bearing minerals, ferrous oxides, respectively.

The applied Abrams ratio (5/7:3/2:4/5) illustrated the hydrothermal altered iron-oxide as green and clay minerals as red colour (figure 3a). Minerals containing iron ions, vegetated zones and hydroxyl minerals show respectively red, green and blue colour using Kaufmann ratio (7/4:4/3:5/7) (figure $3 \mathrm{~b}$ ). Using Chica-Olma ratio (5/7:5/4:3/1), we obtain altered clay minerals as red, iron ions as green and ferrous oxide as blue colour (figure 3c). From these entire analysis maps we found the fumaroles region with high concentrated iron minerals.
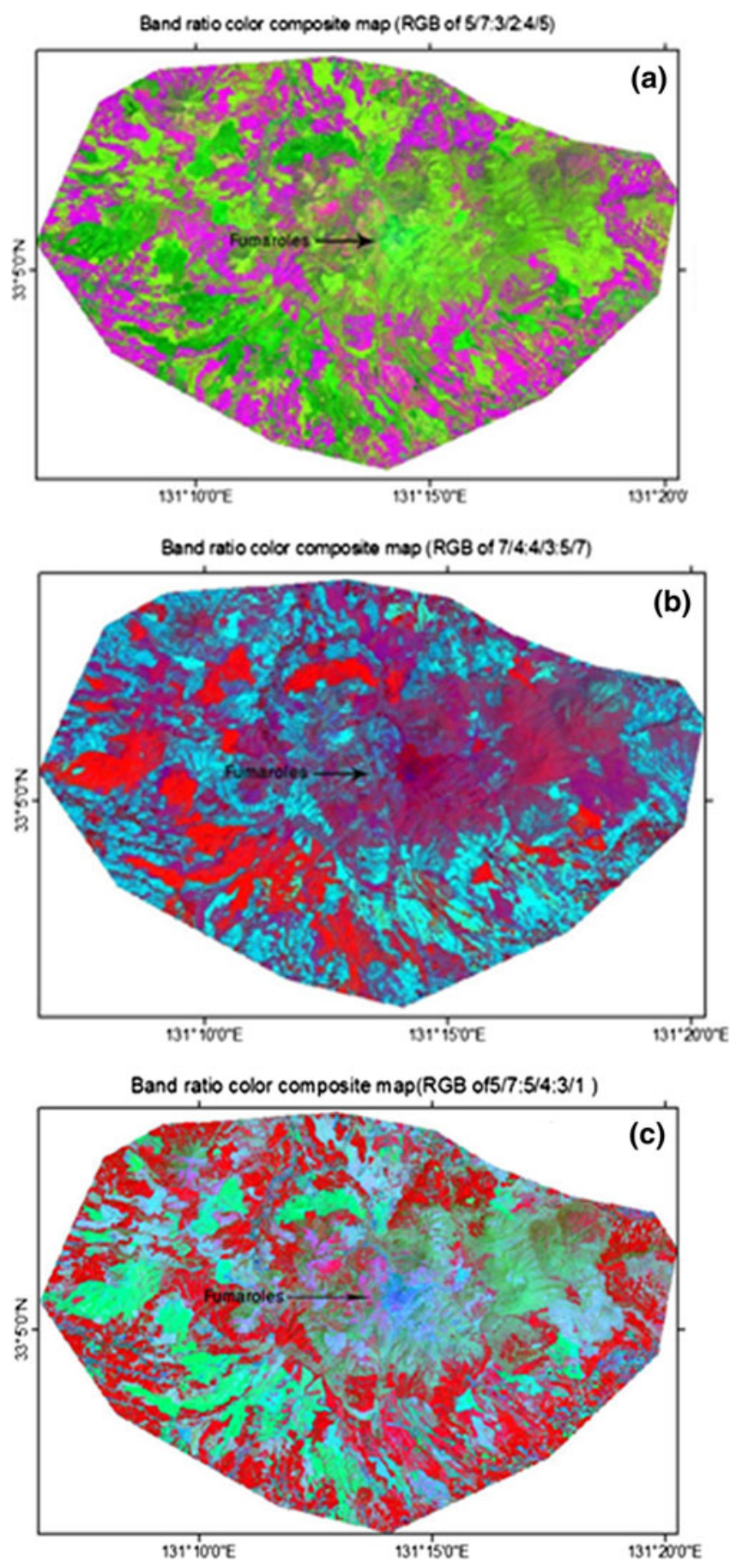

Figure 3. Band ratio analyzed altered mineral deposits: (a) Abrams ratio, (b) Kaufmann ratio and (c) Chica-Olma ratio.

Table 2. Principal component analysis of 7 ETM+ bands of Kuju volcano area.

\begin{tabular}{lcccccccc}
\hline Input band & Band 1 & Band 2 & Band 3 & Band 4 & Band 5 & Band 6 & Band 7 & Eigenvalue $(\%)$ \\
\hline PC 1 & -0.349387 & -0.282127 & -0.307983 & -0.26608 & -0.457903 & -0.560686 & -0.329601 \\
PC 2 & 0.205634 & 0.054379 & -0.223421 & 0.323659 & -0.496991 & 0.527618 & -0.524123 \\
PC 3 & 0.028566 & 0.19669 & 0.190605 & 0.705949 & 0.200251 & -0.524883 & -0.331963 \\
PC 4 & -0.44303 & -0.3822 & -0.555811 & 0.382027 & 0.378048 & 0.2148 & 0.117123 \\
PC 5 & 0.163992 & 0.163111 & -0.275473 & 0.34188 & -0.496108 & -0.193167 & 0.685782 \\
PC 6 & -0.719014 & 0.075001 & 0.536582 & 0.171298 & -0.312635 & 0.219167 & 0.119812 & 0.563 \\
PC 7 & -0.307696 & 0.836931 & -0.382318 & -0.18415 & 0.128219 & 0.016166 & -0.089941 \\
\hline
\end{tabular}


Table 3. Principal component analysis for hydroxyl mapping of Kuju volcano area.

\begin{tabular}{|c|c|c|c|c|c|}
\hline \multirow{2}{*}{$\begin{array}{l}\text { Input } \\
\text { band }\end{array}$} & \multicolumn{4}{|c|}{ Eigenvector loading for hydroxyl mapping } & \multirow{2}{*}{$\begin{array}{c}\text { Eigenvalue } \\
(\%)\end{array}$} \\
\hline & Band 1 & Band 4 & Band 5 & Band 7 & \\
\hline $\mathrm{PC} 1$ & 0.476912 & 0.36369 & 0.64851 & 0.46873 & 94.288 \\
\hline PC 2 & 0.430685 & 0.66533 & 0.31053 & -0.5248 & 4.58571 \\
\hline PC 3 & 0.738561 & 0.51103 & 0.39549 & 0.19225 & 0.98123 \\
\hline $\mathrm{PC} 4$ & 0.203942 & 0.40486 & 0.57148 & 0.68404 & 0.14504 \\
\hline
\end{tabular}

\subsection{Principal component analysis}

The principal components analysis (PCA) uses the principal components transformation technique for reducing dimensionality of correlated multispectral data. The analysis is based on multivariate statistical technique that selects uncorrelated linear combinations (eigenvector loadings) of variables in such a way that each successively extracted linear combination, or principal component (PC), has a smaller variance (Singh and Harrison 1985). The statistical variance in multispectral images is related to the spectral response of various surficial materials such as rocks, soils, and vegetation, and it is also influenced by the statistical dimensionality of the image data (Loughlin 1991). Eigenvector loadings (eigenvalues) give information using magnitude and sign of about which spectral properties of vegetation, rocks and soils are responsible for the statistical variance mapped into each $\mathrm{PC}$, and this is the basis of the Crosta technique.

In our study, the principal component transformation indicates that the first principal component (PC1) is composed of a negative weighing of all total bands (table 2). The PC1 is about $94.594 \%$ of eigenvalue of the total variance for unstretched data PCA. The eigenvector loadings for PC3 indicates that PC3 is dominated by vegetation, which is highly reflective in Band 4; the positive loading of Band 4 in this PC (0.705949) also indicates that strongly vegetated pixels will be bright in this $\mathrm{PC}$ image. The percentage of variance mapped into this 'vegetation' $\mathrm{PC}$ is only $0.973 \%$, which is not a measure of vegetation abundance in the Kuju volcano area, where most pixels will contain some vegetation. Because the eigenvector loadings for Band 1 and Band 3 in PC6 of table 2 are also opposite in sign, we can predict that iron oxides will be distinguished by bright pixels in PC6 of table 1. Hydroxyl-bearing minerals are mapped as dark pixels in PC5 due to the fact that the contribution is negative from Band 5 and positive from Band 7 in this PC5 (table 2). If the number of input channels is reduced to avoid a particular spectral contrast, the chances of defining a unique PC for a specific mineral class will be increased (Loughlin 1991).
The principal components transformation on unstretched Bands 1, 4, 5, and 7 of the Kuju volcano are shown in table 3 . The Bands 2 and 3 have been deliberately omitted to avoid mapping iron oxides, and it should be noted that Band 2 or Band 3 could substitute for Band 1 in this transformation. Following the reasoning process described above, we can predict that PC1 is the 'albedo' image, PC2 describes the contrast between the short wave infrared (SWIR) and the visible region, PC3 is darkest for vegetation, and $\mathrm{PC} 4$ highlights hydroxyl-bearing minerals as dark pixels. This 'hydroxyl' (H) image is therefore negated in figure 4 to show anomalous concentrations of $\mathrm{H}$ as brightest zones. The methodology for hydroxyl mapping by PCA on Bands 1, 4, 5 and 7 is to examine the eigenvector loadings for Bands 5 and 7 , in the PC3 and PC4 images. The PC image that best discriminates hydroxyl-bearing minerals is that with a high or moderate eignvector loading, irrespective of sign, for Band 7 and a high or moderate eignevector loading of opposite sign for Band 5. Negation of those PCs in which the Band 7 loading is positive makes the anomalous pixels brightest in all cases (figure 4). PC4 in table 2 with a relatively strong positive loading for Band 7

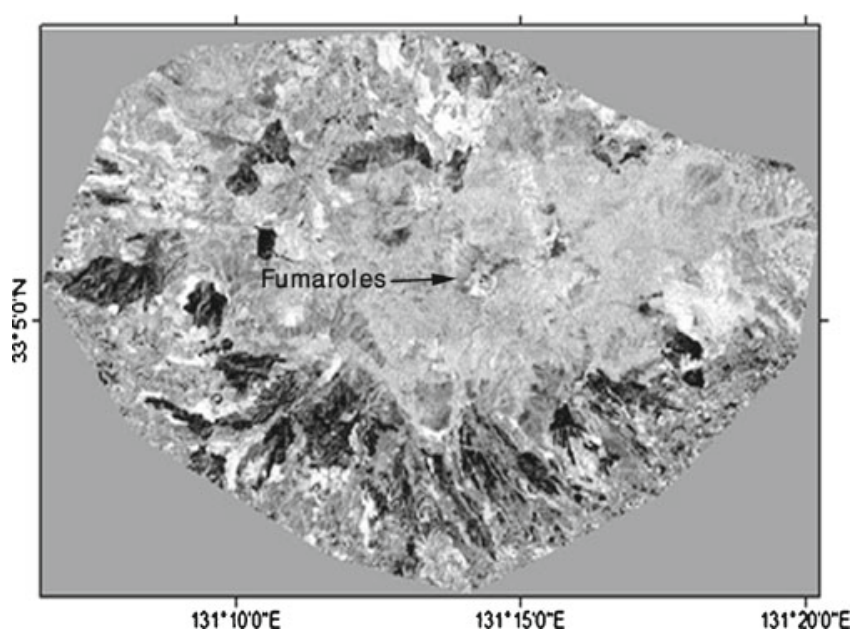

Figure 4. PC4, bright pixels represent the hydroxyl minerals. 
Table 4. Principal component analysis for iron-oxide mapping of Kuju volcano area.

\begin{tabular}{|c|c|c|c|c|c|}
\hline \multirow{2}{*}{$\begin{array}{l}\text { Input } \\
\text { band }\end{array}$} & \multicolumn{4}{|c|}{ Eigenvector loading for iron-oxide mapping } & \multirow{2}{*}{$\begin{array}{c}\text { Eigenvalue } \\
(\%)\end{array}$} \\
\hline & Band 1 & Band 3 & Band 4 & Band 5 & \\
\hline PC 1 & 0.488797 & 0.43895 & 0.375668 & 0.653662 & 95.27212 \\
\hline PC 2 & 0.406451 & -0.203751 & 0.689737 & -0.56351 & 3.303728 \\
\hline PC 3 & -0.597175 & -0.411839 & 0.5587 & 0.402025 & 1.044604 \\
\hline $\mathrm{PC} 4$ & 0.489139 & -0.772138 & -0.266442 & 0.305867 & 0.279553 \\
\hline
\end{tabular}

(0.684039) and moderate negative loading for Band $5(-0.57148)$ can be considered as an ' $\mathrm{H}$ ' image for the Kuju volcano area.

The principal components transformation on unstretched Bands 1, 3, 4 and 5 of the study area is present in table 4 . The PCs can be interpreted as albedo in $\mathrm{PC} 1$, vegetation in $\mathrm{PC} 2$, IR versus visible in $\mathrm{PC} 3$, and iron oxide as dark pixels in PC4 (eigenvector loading for Band $3=-0.772138$ and for Band $1=+0.489139)$. This PC image (F) can be negated to show iron oxide stained areas as bright pixels (figure 5). The rules for iron oxide mapping are similar to those for hydroxyl mapping.

\subsection{Iron-oxide and hydroxyl mapping using Crosta technique}

For applying Crosta techniques, the information stored on the hydroxyl $(\mathrm{H})$ and iron-oxide $(\mathrm{F})$ images are combined to produce a map displaying the pixels with anomalous concentrations of both hydroxyls and iron-oxides as the brightest. This new image is called $\mathrm{H}+\mathrm{F}$ image. This merging of the two images is also achieved by PCA; such as the principal component having positive eigenvalues from both input bands is selected. By using the Crosta $\mathrm{H}, \mathrm{H}+\mathrm{F}$ and $\mathrm{F}$ images, where they are stretched in desired combinations, colour composites are prepared. Respectively, display of

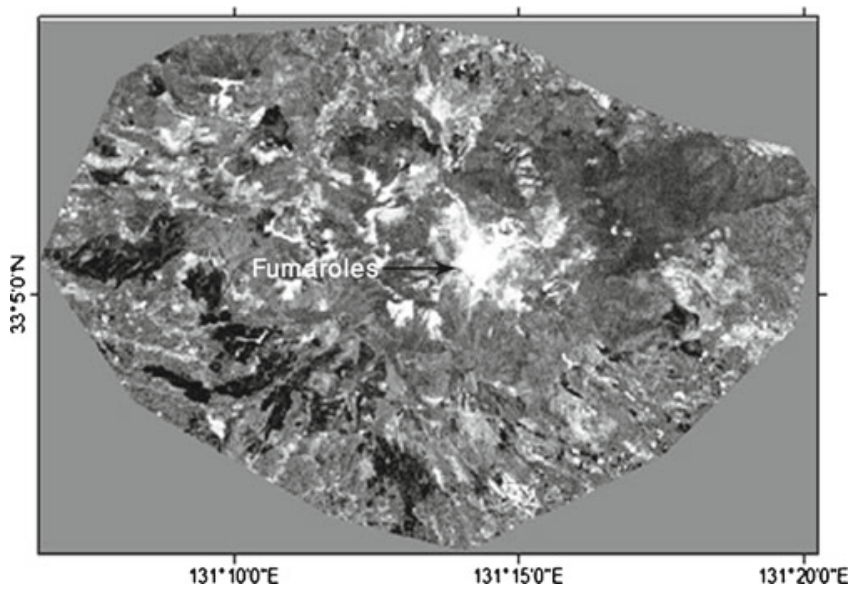

Figure 5. PC4, bright pixels represent iron-oxide zone. the Crosta images of $\mathrm{H}, \mathrm{H}+\mathrm{F}$ and $\mathrm{F}$ in RGB channels returns a dark bluish colour composite image on which alteration zones are unusually bright (figure 6). White pixels within alteration zones are the areas where both iron-stained and argillized. Bright reddish to orange zones are more argillaceous than iron-stained; and bright cyan to bluish zones are more iron-stained than argillized (Loughlin 1991). It is clear from this principal component analysis maps that the altered iron-oxide deposits are abundant near the fumaroles of this volcano.

\subsection{Least square fitting method}

Least square fitting method (Ls-Fit) performs a linear band prediction using least-squares fitting. It is the task to find regions of anomalous spectral response in a dataset. It calculates the covariance of the input data and is used to predict the selected band as a linear combination of the predictor bands plus an offset. The difference (residual) between the actual band and the modelled band is calculated and output as an image. Both Ls-Fit and Crosta methods were performed on the data to map spectral signatures of hydrothermal alterations (i.e., clay minerals and iron oxides), while LS-Fit method performs a linear band prediction using a least square fitting technique. The

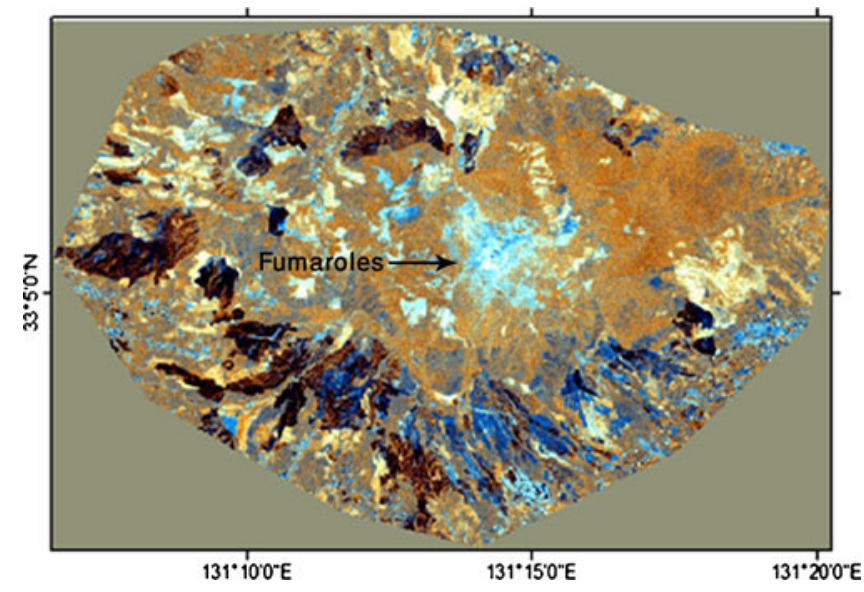

Figure 6. Hydrothermal alteration mapping using Crosta techniques (H: H+F: F as R: G: B). 
distribution of clay alterations illustrates as dark pixels produced using LS-Fit model (figure 7a). This map was created by using all the six visible and short wave infrared (SWIR) bands as the input bands and Band 7 as the modelled band. In addition, since the Fe-oxidation is usually associated with hydrothermal alteration, the ETM+ data of
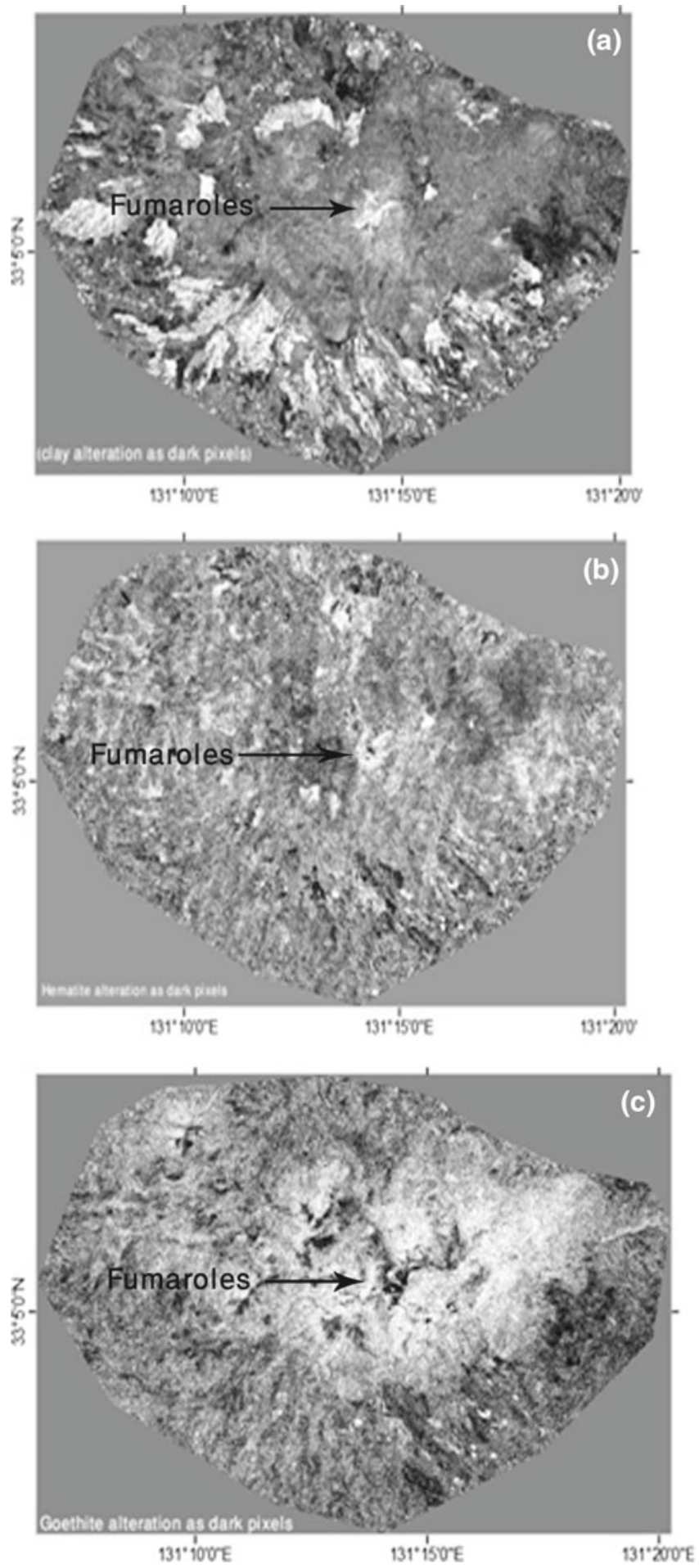

Figure 7. Ls-fit images showing as bright pixels: (a) clay alterations, (b) hematite alterations and (c) goethite alterations.

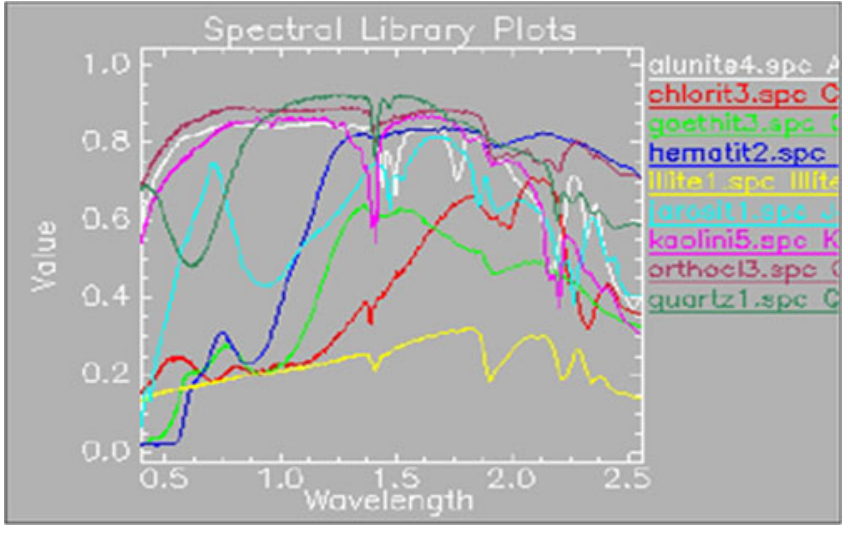

Figure 8. USGS library mineral spectra used in this study.

the Kuju volcano area were also processed to map Fe-oxides, by using Ls-Fit method. In this case, the ferrous Fe-oxides show an absorption feature across Band 3; therefore, this band was used as a model band to map iron-oxides (e.g., hematite) (figure 7b). By using residual Band 1, we created a map showing goethite alteration as dark pixels (figure $7 \mathrm{c}$ ). The fumaroles area is concentrated with iron-oxide minerals mostly goethite and hematite in this map. We used only the visible and IR bands to calculate the residuals of these bands.

\subsection{Hydrothermal altered minerals mapping by spectral reflectance analysis}

The target detection wizard analysis technique of ENVI 4.3 was applied to detect the hydrothermal altered minerals using USGS library mineral spectra (figure 8) in this study. This method consists of 10 data processing steps including atmospheric correction, MNF transform, selecting the target using defined library spectra, filtering the target using match filtering method and finally export result with statistics. The study performed analyses including the processing and filtering of selected alteration types of the minerals which are kaolinite, illite, alunite, orthoclase, quartz, chlorite, hematite, goethite and jarosite (figure 9 ). The matched filtering (MF) finds the abundance of targets using a partial unmixing algorithm (Jin et al. 2009). This technique maximizes the response of the known spectra and suppresses the response of the composite unknown background, therefore matching the known signature. It provides a rapid means of detecting specific materials based on matching with target spectra and does not require knowledge of all the endmembers within an image scene. The applied filtering threshold value was selected as 0.50 .

The oxidation type of alteration (goethite, hematite and jarosite) showed more concentration 
around the fumarolic region of the study area (figure 9c). Figure 9(a) showed that the hydrothermally altered argillaceous minerals (i.e., alunite, illite and kaolinite) were abundant within the fumaroles zone of our study area. The potassic, prophylitic and silicific types of alterations of
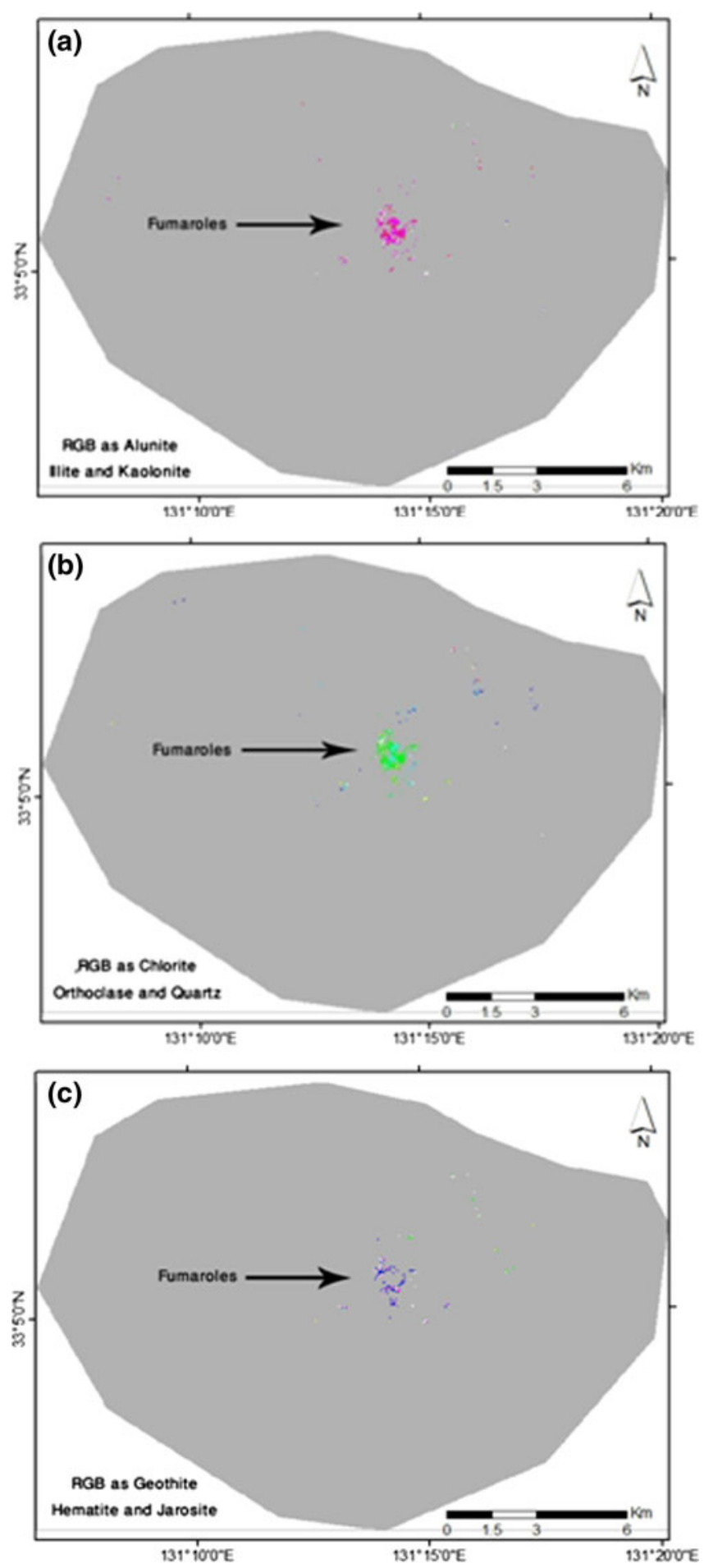

Figure 9. Reference spectra analysis altered minerals deposit: (a) Alunite, Illite and Kaolinite; (b) Chlorite, Orthoclase and Quartz; (c) Goethite, Hematite and Jarosite. the study area are also concentrated within the fumaroles (figure 9b).

\section{Conclusions}

After using the conventional alteration mapping methods on the Landsat image in the study area, we found that the colour composite and band ratio methods showed their efficiency to define the area of hydrothermal alteration. Principal component analysis illustrated the iron-oxides and hydroxyl altered minerals area of this region very clearly. The altered iron-oxide deposits were found more concentrated around the fumaroles in the Crosta analyzed map. We have mapped successfully the spatial distribution of goethite, hematite and clay minerals of our study area using the least square method. ENVI target detection wizard approach showed a very efficient tool to identify the specific hydrothermal altered minerals using USGS library mineral spectra in this study. In conclusion, we found all of the hydrothermally altered minerals in and around the fumarole area of Kuju volcano. So, it is quite clear that all these methods are quite efficient to delineate hydrothermal alteration products using Landsat 7 ETM+ image in and around Kuju volcano area.

\section{Acknowledgements}

The authors would like to show their sincere gratitude to G-COE of Kyushu University for giving financial support for this research. They also acknowledged NASA warehouse inventory for providing satellite image with free of cost in this study. The authors would also like to thank the people of Geothermics Laboratory of Kyushu University for their support. They are grateful to Dr Somnath Dasgupta who has reviewed, corrected and logically commented to upgrade the manuscript.

\section{References}

Abdelsalam M G, Stern R J and Berhane W G 2000 Mapping gossans in arid regions with Landsat TM and SIR-C images: The Beddaho alteration zone in northern Eritrea; J. African Earth Sci. 30(4) 903-916.

Beane R E 1982 Hydrothermal alteration in silicate rocks, southwestern North America; In: Advances in Geology of the Porphyry Copper Deposits, Southwestern North America: Tucson (ed.) Titley S R, Univ. Ariz. Press, Chapter 6.

Crosta A, Moore J McM 1989 Enhancement of Landsat Thematic Mapper imagery for residual soil mapping in SW Minais Gerais State, Brazil: A prospecting case history in Greenstone belt terrain; International Proceedings of the Seventh Erim Thematic Conference: Remote Sensing for Exploration Geology, pp. 1173-1187. 
Goetz A F, Rock F H and Rowan B N 1983 Remote sensing for exploration: An overview; Eco. Geol. 78 573-590.

Hunt G R 1979 Near Infrared (1.3-2.4 $\mu \mathrm{m})$ spectra of alteration minerals - potential for use in remote sensing; Geophysics 44 1974-1986.

Hunt G R 1980 Electromagnetic radiation - the communication link in remote sensing; In: Remote Sensing in Geology (eds) Siegal B S and Gillespie A R (New York: Wiley).

Hunt G R and Ashley R P 1979 Spectra of altered rocks in the visible and near infrared; Econ. Geol. 74 1613-1629.

Jensen J R 1996 Introductory digital image processing: A remote sensing perspective; 2nd edn, Prentice Hall Series in Geographic Information Science; 318p.

Jin X, Paswaters S and Cline H 2009 A comparative study of target detection algorithms for hyperspectral imagery; In: Algorithms and Technologies for Multispectral, Hyperspectral, and Ultraspectral Imagery XV, Proceedings of SPIE $\mathbf{7 3 3 4} 73341 \mathrm{~W} 1-73341 \mathrm{~W} 12$.

Kamata H and Kodama K 1994 Tectonics of an arc-arc junction: An example from Kyushu Island at the junction of the Southwest Japan Arc and the Ryukyu Arc; Tectonophys. 233 69-81.

Kamata H, Hoshizumi H, Kawanabe Y, Ito J, Watanabe K and Yokoyama S 1996 Geology of small-scale pyroclastic flow deposits and a high-temperature debris-avalanche deposit from Kuju volcano, Japan; Volcanol. Soc. Japan (Abstract) $\mathbf{2} 121$.

Karnieli A, Ben-Dor E, Bayarjargal Y and Lugas R 2004 Radiometric saturation of Landsat-7 ETM+data over the Negev Desert: Problems and solutions; Int. J. Appl. Earth Obs. Geoinf. 5 219-237.

Lillesand T M, Kiefer R W and Chipman J W 2004 Remote sensing and image interpretation; 5th edn, John Wiley and Sons Inc., New York, ISBN 0-471-25515-7, 763p.

Loughlin W P 1991 Principal component analysis for alteration mapping; Photogrammetric Eng. Rem. Sens. 57(g) 1163-1169.

Madani A, Abdel Rahman E M, Fawzy K M and Emam A 2003 Mapping of the hydrothermal alteration zones at Haimur Gold Mine Area, South Eastern Desert, Egypt using remote sensing techniques; The Egyptian J. Rem. Sens. Space Sci. 6 47-60.

Markham B L, Dabney P W, Storey J C, Morfitt R, Knight E J, Kvaran G and Lee K 2008 Landsat Data Continuity Mission Calibration and Validation; ASPRS Publications, Colorado, USA.

Mather P M 1987 Computer Processing of Remotely Sensed Images: An Introduction; John Wiley and Sons; ISBN: 0-471-9064-4.

Poormirzaee R and Oskouei M M 2009 Detection minerals by advanced spectral analysis in ETM+ imagery; Proceeding of 7th Iranian Student Conference Mining Engineering, Tabriz, pp. 111-119.

Ramadan T M, Abdelsalam M G and Stern R J 2001 Mapping gold-bearing massive sulfide deposits in the neoproterozoic Allaqi Suture, Southeast Egypt with Landsat TM and SIR-C/X SAR images; Photogrammetric Eng. Rem. Sens. 67(4) 491-497.

Ramadan T M and Kontny A 2004 Mineralogical and structural characterization of alteration zones detected by orbital remote sensing at Shalatein District, SE Desert, Egypt; J. African Earth Sci. 40 89-99.

Rowan L C 1983 Near-infrared iron absorption bands: Applications to geologic mapping and mineral exploration; In: Remote sensing (eds) Watson and Regan; Society of Exploration in Geophysicists, Reprint Series 3 250-268.

Segal D B 1983 Use of Landsat multispectral scanner data for definition of Limonitic exposures in heavily vegetated areas, EL Paso, Texas; Econ. Geol. 78 711-722.

Singh A and Harrison A 1985 Standardized principal components; Int. J. Rem. Sens. 6(6) 883-896.

Taranik D L, Kruse F A, Goetz A F H and Atkinson W W 1991 Remote sensing of ferric iron minerals as guides for gold exploration; Proceedings Eighth Thematic Conference on Geologic Remote Sensing, Denver, Colorado, pp. 197-228.

Yetkin E 2003 Alteration Mapping by Remote Sensing: Application to Hasanda-Melendiz Volcanic Complex, M.Sc. Thesis, Middle East Technical University, Ankara, Turkey, $97 \mathrm{p}$. 